\title{
Observation of $Z>2$ trapped nuclei by AMS on ISS
}

\section{Francesca Giovacchini, ${ }^{a}$ Alberto Oliva ${ }^{b}$ and Martha Valencia-Otero ${ }^{c, *}$ on behalf of the AMS Collaboration}

(a complete list of authors can be found at the end of the proceedings)

${ }^{a}$ Centro de Investigaciones Energéticas, Medioambientales y Tecnológicas,

Av. Complutense, 40, 28040, Madrid, Spain.

${ }^{b}$ Istituto Nazionale di Fisica Nucleare Sezione di Bologna,

Viale Carlo Berti Pichat 6/2, 40127, Bologna, Italy.

${ }^{c}$ National Central University,

No.300, Zhongda road, Zhongli district, Taoyuan, Taiwan.

E-mail: Francesca.Giovacchini@cern.ch, alberto.oliva@bo.infn.it,

martha.liliana.valencia.otero@cern.ch

The Alpha Magnetic Spectrometer (AMS-02) is a high energy particle physics experiment operating continuously aboard the International Space Station (ISS) since the 19th of May of 2011. A component of trapped $Z>2$ ions located in the South Atlantic Anomaly (SAA) has been detected traversing the instrument both in down-going and up-going directions.

$37^{\text {th }}$ International Cosmic Ray Conference (ICRC 2021)

July 12th-23rd, 2021

Online - Berlin, Germany

\footnotetext{
*Presenter
} 
The Alpha Magnetic Spectrometer (AMS-02) particle detector, launched in 2011 and installed as a module in the International Space Station (ISS), has the potential of precisely measuring cosmic ray fluxes and composition, including ions up to $Z=26$. Since the beginning of its operation, AMS-02 has collected over 150 billion events. In this paper we report the observation of a $Z>2$ trapped nuclei population, detected near the equator inside the SAA, in 8.5 years of data collection.

\section{Data Sample}

The selection considers the widest field of view of the detector, up to 45 degrees with respect to vertical, and includes events traversing AMS in both, down-going and up-going directions. A layout of the detector is presented in [1-2]. Events are required to have a good track reconstruction in the Inner Tracker and satisfy good quality criteria on velocity and charge reconstruction. Charge measurements in the Inner Tracker and Time of Flight (TOF) are selected to be in accordance with $Z>2$ ions and conditions to reject interacting events inside the detector have been implemented as well. This study focuses in the equatorial region with $\left|\Theta_{M}\right|<0.3$ and velocity $|\beta|<0.86$.

\section{Backtracing Procedure}

The procedure of backtracing a charged particle's trajectory to ascertain its origin, consists on propagating the particle with inverted sign an arrival direction in the Earth's magnetic field. In this study, we have applied a backtracing routine that implements the IGRF-13 geomagnetic field model [3]. The outcome of the procedure classifies events in the following possible categories [4]:

- Cosmic: when the particle trajectory escapes a limit of 25 Earth's radii.

- Quasi Trapped (QT): if the particle reaches a boundary set at $40 \mathrm{~km}$ from the Earth's surface.

- Stable Trapped (ST): if after $50 \mathrm{~s}$ of propagation, the backtracing outcome is neither cosmic nor quasi trapped.

However, it is important to consider two effects that could introduce a difference in the particle classification when the backtracing is performed once. Firstly, the existence of the so-called penumbra region, where small variations of the initial conditions could lead to a change of the procedure's outcome. Secondly, uncertainties in the backtracing parameters, such as the rigidity determination, the ISS orbit location and AMS direction could also modify the particle categorization. Therefore, in order to account for these effects, the backtracing routine was conducted several times, varying the particle arrival direction $\left( \pm 0.2^{\circ}\right)$ and space station position in time $( \pm 50 \mathrm{~ms})$. Finally, the previously described procedure allows a conservative classification of our data sample and the selection of those particles with QT and ST trajectories is certainly achieved.

\section{Results}

Succeeding our sample selection and backtracing procedure, a population of trapped ions has been identified in the equatorial region inside the SAA, as shown in Fig.1. Both, down-going and up-going events are included in the observation and these two populations present similar relative 


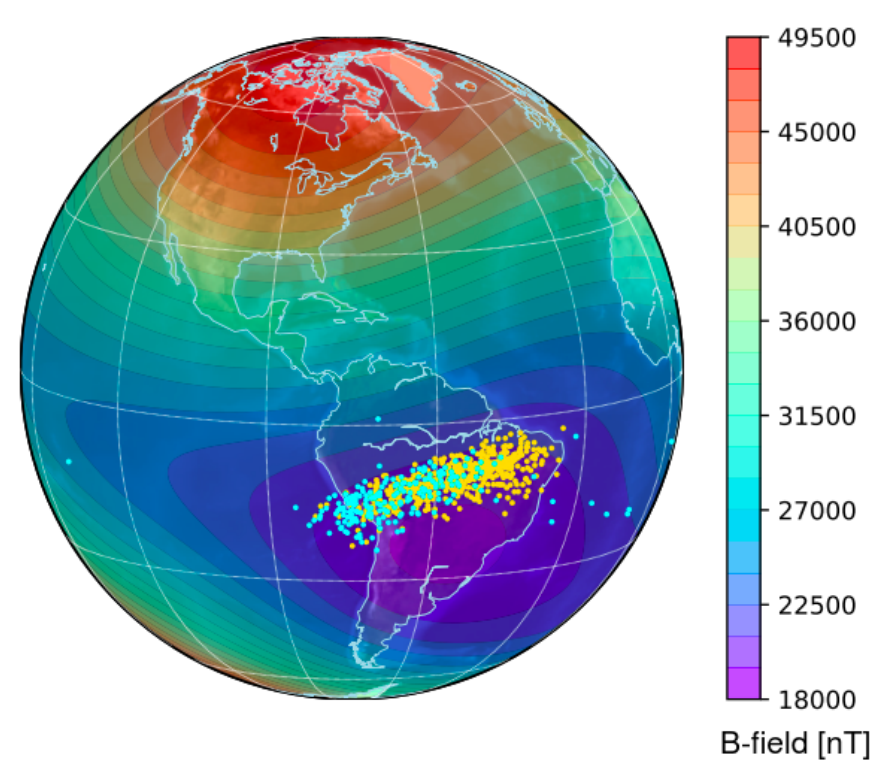

Figure 1: Trapped nuclei events detection location. Down-going and up-going particles are displayed in cyan and yellow respectively, on top of the total Earth's magnetic field map [nT].

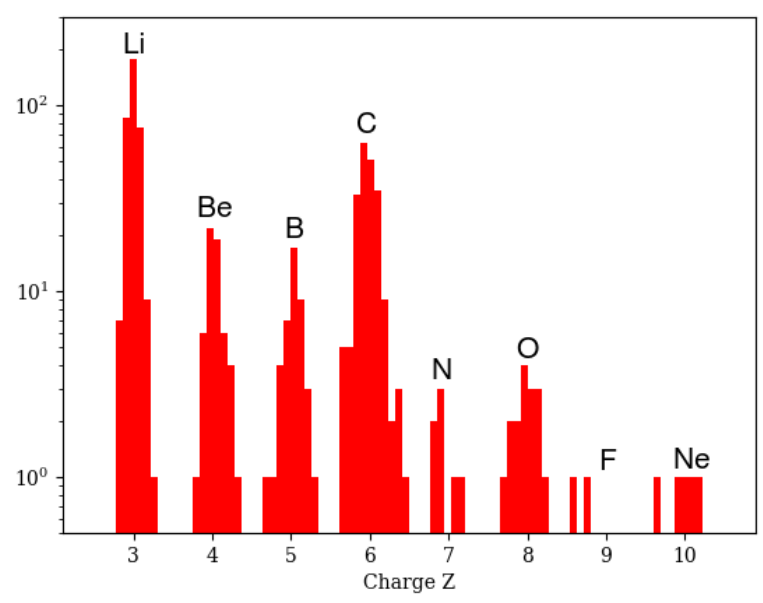

Figure 2: Charge distribution of $Z>2$ trapped ions. Both, down-going and up-going events are included.

abundances. The charge distribution of trapped ions is depicted in Fig.2, where an overabundance of Lithium and Carbon is noticeable. In addition, the $\mathrm{Li} / \mathrm{C}$ and $\mathrm{C} / \mathrm{O}$ ratios for this trapped population are remarkably different from those observed in galactic cosmic rays (where $\mathrm{Li} / \mathrm{C} \sim 0.1$ and $\mathrm{C} / \mathrm{O}$ $\sim 1)$.

\section{Conclusions}

A search of trapped nuclei with $Z>2$ has been carried out with 8.5 years of AMS data collection. By means of a backtracing procedure, the classification of events as trapped has been conservatively 
achieved. Hence, we report the observation of a population of $Z>2$ trapped nuclei, near the equator inside the SAA, including events entering AMS from down-going and up-going directions. Our results show that the relative abundances of the trapped particles are distinctly different from the galactic cosmic rays. This study represents the first observation of $Z>2$ trapped ions above $1 \mathrm{GV}$.

\section{References}

[1] Battiston, R., The antimatter spectrometer (AMS-02): A particle physics detector in space, Nuclear Instruments and Methods in Physics Research Section A: Accelerators, Spectrometers, Detectors, and Associated Equipment, Vol. 588, (2008).

[2] Kounine. A. The Alpha Magnetic Spectrometer on the International Space Station, International Journal of Modern Physics, E 21.08 (2012).

[3] Thébault, E. et al., International geomagnetic reference field: the 12th generation, Earth, Planets and Space, 67(1), (2015).

[4] Fiandrini, E. et al., Protons with kinetic energy E $>70 \mathrm{MeV}$ trapped in the Earth's radiations belts, Journal of Geophysical Research, 109, (2014). 


\section{Full Authors List: AMS Collaboration}

M. Aguilar, ${ }^{30}$ L. Ali Cavasonza, ${ }^{1}$ M. S. Allen, ${ }^{10}$ B. Alpat,${ }^{37}$ G. Ambrosi, ${ }^{37}$ L. Arruda,${ }^{28}$ N. Attig, ${ }^{24}$ F. Barao, ${ }^{28}$ L. Barrin, ${ }^{15}$ A Bartoloni, ${ }^{43}$ S. Başeğmez-du Pree, ${ }^{18, *}$ R. Battiston, ${ }^{40,41}$ M. Behlmann, ${ }^{10}$ B. Beranek, ${ }^{1}$ J. Berdugo, ${ }^{30}$ B. Bertucci, ${ }^{37,38}$ V. Bindi, ${ }^{20}$ K. Bollweg, ${ }^{21}$ B. Borgia, ${ }^{43,44}$ M. J. Boschini, ${ }^{32}$ M. Bourquin, ${ }^{16}$ E. F. Bueno, ${ }^{18}$ J. Burger, ${ }^{10}$ W. J. Burger ${ }^{40}$ S. Burmeister, ${ }^{26}$ X. D. Cai, ${ }^{10}$ M. Capell, ${ }^{10}$ J. Casaus, ${ }^{30}$ G. Castellini, ${ }^{14}$ F. Cervelli, ${ }^{39}$ Y. H. Chang, ${ }^{48,49}$ G. M. Chen, ${ }^{6,7}$ G. R. Chen, ${ }^{23}$ H. S. Chen,,${ }^{6,7}$ Y. Chen, ${ }^{16,23}$ L. Cheng, ${ }^{23}$ H. Y. Chou, ${ }^{49}$ S. Chouridou, ${ }^{1}$ V. Choutko, ${ }^{10}$ C. H. Chung, ${ }^{1}$ C. Clark, ${ }^{10,21}$ G. Coignet, ${ }^{3}$ C. Consolandi, ${ }^{20}$ A. Contin, ${ }^{8,9}$ C. Corti, ${ }^{20}$ Z. Cui, ${ }^{22,23}$ K. Dadzie, ${ }^{10}$ C. Delgado, ${ }^{30}$ S. Della Torre, ${ }^{32}$ M. B. Demirköz, ${ }^{2}$ L. Derome, ${ }^{17}$ S. Di Falco, ${ }^{39}$ V. Di Felice, ${ }^{45}$ C. Díaz, ${ }^{30}$ F. Dimiccoli, ${ }^{40}$ P. von Doetinchem, ${ }^{20}$ F. Dong, ${ }^{35}$ F. Donnini, ${ }^{45}$, M. Duranti, ${ }^{37}$ A. Egorov, ${ }^{10}$ A. Eline, ${ }^{10}$ J. Feng, ${ }^{10}$ E. Fiandrini, ${ }^{37,38}$ P. Fisher, ${ }^{10}$ V. Formato, ${ }^{45}$ C. Freeman, ${ }^{20}$ Y. Galaktionov, ${ }^{10}$ C. Gámez, ${ }^{30}$ R. J. García-López,${ }^{27}$ C. Gargiulo, ${ }^{15} \mathrm{H}$. Gast, ${ }^{1}$ M. Gervasi, ${ }^{32,33}$ F. Giovacchini, ${ }^{30}$ D. M. Gómez-Coral, ${ }^{20}$ J. Gong, ${ }^{35}$ C. Goy, ${ }^{3}$ V. Grabski, ${ }^{31}$ D. Grandi, ${ }^{32,33}$ M. Graziani, ${ }^{37,38}$ S. Haino, ${ }^{48}$ K. C. Han, ${ }^{29}$ R. K. Hashmani, ${ }^{2}$ Z. H. He, ${ }^{19}$ B. Heber, ${ }^{26}$ T. H. Hsieh, ${ }^{10}$ J. Y. Hu, ${ }^{6,7}$ M. Incagli, ${ }^{39}$ W. Y. Jang, ${ }^{13}$ Yi Jia, ${ }^{10}$ H. Jinchi, ${ }^{29}$ K. Kanishev, ${ }^{40}$ B. Khiali, ${ }^{45}$, G. N. Kim, ${ }^{13}$ Th. Kirn, ${ }^{1}$ M. Konyushikhin, ${ }^{10}$ O. Kounina, ${ }^{10}$ A. Kounine,,${ }^{10}$ V. Koutsenko, ${ }^{10}$ A. Kuhlman, ${ }^{20}$ A. Kulemzin, ${ }^{10}$ G. La Vacca, ${ }^{32,33}$ E. Laudi, ${ }^{15}$ G. Laurenti, ${ }^{8}$ I. Lazzizzera, ${ }^{40,41}$ A. Lebedev, ${ }^{10}$ H. T. Lee, ${ }^{47}$ S. C. Lee, ${ }^{48}$ J. Q. Li, ${ }^{35}$ M. Li, ${ }^{1}$ Q. Li, ${ }^{35}$ S. Li, ${ }^{1}$ J. H. Li, ${ }^{22}$ Z. H. Li, ${ }^{6,7}$ J. Liang, ${ }^{22}$ C. Light, ${ }^{20}$ C. H. Lin,,${ }^{48}$ T. Lippert, ${ }^{24}$ J. H. Liu, ${ }^{5}$ Z. Liu, ${ }^{16}$ S. Q. Lu, ${ }^{48}$ Y. S. Lu, ${ }^{6}$ K. Luebelsmeyer, ${ }^{1}$ J. Z. Luo, ${ }^{35}$ Xi Luo, ${ }^{23}$ S. S. Lyu, ${ }^{19}$ F. Machate, ${ }^{1}$ C. Mañá, ${ }^{30}$ J. Marín, ${ }^{30}$ J. Marquardt, ${ }^{26}$ T. Martin, ${ }^{10,21}$ G. Martínez, ${ }^{30}$ N. Masi, ${ }^{8,9}$ D. Maurin, ${ }^{17}$ A. Menchaca-Rocha, ${ }^{31}$ Q. Meng, ${ }^{35}$ V. V. Mikhailov, ${ }^{34}$ D. C. Mo, ${ }^{19}$ M. Molero, ${ }^{30}$ P. Mott, ${ }^{10,21}$ L. Mussolin, ${ }^{37,38}$ J. Negrete, ${ }^{20}$ N. Nikonov, ${ }^{1}$ F. Nozzoli ${ }^{40}$ A. Oliva,${ }^{8}$ M. Orcinha, ${ }^{28}$ M. Palermo,${ }^{20}$ F. Palmonari, ${ }^{8,9}$ M. Paniccia, ${ }^{16}$ A. Pashnin, ${ }^{10}$ M. Pauluzzi ${ }^{37,38}$ S. Pensotti, ${ }^{32,33}$ H. D. Phan,,${ }^{10}$ R. Piandani, ${ }^{25}$ V. Plyaskin, ${ }^{10}$ S. Poluianov,,${ }^{36}$ X. Qin, ${ }^{10}$ Z. Y. Qu, ${ }^{48}$ L. Quadrani, ${ }^{8,9}$ P. G. Rancoita, ${ }^{32}$ D. Rapin, ${ }^{16}$ A. Reina Conde, ${ }^{27}$ E. Robyn, ${ }^{16}$ S. Rosier-Lees, ${ }^{3}$ A. Rozhkov, ${ }^{10}$ D. Rozza, ${ }^{32,33}$ R. Sagdeev, ${ }^{11}$ S. Schael, ${ }^{1}$ A. Schulz von Dratzig, ${ }^{1}$ G. Schwering, ${ }^{1}$ E. S. Seo, ${ }^{12}$ Z. Shakfa, ${ }^{2}$ B. S. Shan, ${ }^{4}$ T. Siedenburg, ${ }^{1}$ J. W. Song, ${ }^{22}$ X. J. Song, ${ }^{23}$ R. Sonnabend, ${ }^{1}$ L. Strigari, ${ }^{43}$ T. Su, ${ }^{23}$ Q. Sun, ${ }^{22}$ Z. T. Sun, ${ }^{6,7}$ M. Tacconi, ${ }^{32,33}$ X. W. Tang, ${ }^{6}$ Z. C. Tang,,${ }^{6}$ J. Tian, ${ }^{37,38}$ Samuel C. C. Ting, ${ }^{10,15}$ S. M. Ting, ${ }^{10}$ N. Tomassetti,,${ }^{37,38}$ J. Torsti, ${ }^{50}$ C. Tüysüz, ${ }^{2}$ T. Urban,,${ }^{10,21}$ I. Usoskin, ${ }^{36}$ V. Vagelli, ${ }^{42,37}$ R. Vainio,,${ }^{50}$ M. Valencia-Otero, ${ }^{49}$ E. Valente, ${ }^{43,44}$ E. Valtonen, ${ }^{50}$ M. Vázquez Acosta, ${ }^{27} \mathrm{M}$. Vecchi, ${ }^{18}$ M. Velasco, ${ }^{30}$ J. P. Vialle, ${ }^{3}$ C. X. Wang, ${ }^{22}$ L. Wang, ${ }^{5}$ L. Q. Wang, ${ }^{22}$ N. H. Wang, ${ }^{22}$ Q. L. Wang, ${ }^{5}$ S. Wang, ${ }^{20}$ X. Wang, ${ }^{10}$ Yu Wang, ${ }^{22}$ Z. M. Wang, ${ }^{23}$ J. Wei, ${ }^{16}$ Z. L. Weng, ${ }^{10}$ H. Wu ${ }^{35}$ R. Q. Xiong, ${ }^{35}$ W. Xu, ${ }^{22,23}$ Q. Yan, ${ }^{10}$ Y. Yang, ${ }^{46}$ I. I. Yashin, ${ }^{34}$ H. Yi, ${ }^{35}$ Y. M. Yu, ${ }^{19}$ Z. Q. Yu, ${ }^{6}$ M. Zannoni, ${ }^{32,33}$ C. Zhang, ${ }^{6}$ F. Zhang, ${ }^{6}$ F. Z. Zhang, ${ }^{6,7}$ J. H. Zhang, ${ }^{35}$ Z. Zhang, ${ }^{10}$ F. Zhao, ${ }^{6,7}$ C. Zheng, ${ }^{23}$ Z. M. Zheng, ${ }^{4}$ H. L. Zhuang, ${ }^{6}$ V. Zhukov, ${ }^{1}$ A. Zichichi, ${ }^{8,9}$ and P. Zuccon ${ }^{40,41}$

${ }^{1}$ I. Physics Institute and JARA-FAME, RWTH Aachen University, 52056 Aachen, Germany

${ }^{2}$ Department of Physics, Middle East Technical University (METU), 06800 Ankara, Turkey

${ }^{3}$ Université Grenoble Alpes, Université Savoie Mont Blanc, CNRS, LAPP-IN2P3, 74000 Annecy, France

${ }^{4}$ Beihang University (BUAA), Beijing, 100191, China

${ }^{5}$ Institute of Electrical Engineering (IEE), Chinese Academy of Sciences, Beijing, 100190, China

${ }^{6}$ Institute of High Energy Physics (IHEP), Chinese Academy of Sciences, Beijing, 100049, China

${ }^{7}$ University of Chinese Academy of Sciences (UCAS), Beijing, 100049, China

${ }^{8}$ INFN Sezione di Bologna, 40126 Bologna, Italy

${ }^{9}$ Universitá di Bologna, 40126 Bologna, Italy

${ }^{10}$ Massachusetts Institute of Technology (MIT), Cambridge, Massachusetts 02139, USA

${ }^{11}$ East-West Center for Space Science, University of Maryland, College Park, Maryland 20742, USA

${ }^{12}$ IPST, University of Maryland, College Park, Maryland 20742, USA

${ }^{13}$ CHEP, Kyungpook National University, 41566 Daegu, Korea

${ }^{14}$ CNR-IROE, 50125 Firenze, Italy

${ }^{15}$ European Organization for Nuclear Research (CERN), 1211 Geneva 23, Switzerland

${ }^{16}$ DPNC, Université de Genève, 1211 Genève 4 , Switzerland

${ }^{17}$ Université Grenoble Alpes, CNRS, Grenoble INP, LPSC-IN2P3, 38000 Grenoble, France

${ }^{18}$ Kapteyn Astronomical Institute, University of Groningen, P.O. Box 800, 9700 AV Groningen, Netherlands

${ }^{19}$ Sun Yat-Sen University (SYSU), Guangzhou, 510275, China

${ }^{20}$ Physics and Astronomy Department, University of Hawaii, Honolulu, Hawaii 96822, USA

${ }^{21}$ National Aeronautics and Space Administration Johnson Space Center (JSC), Houston, Texas 77058, USA

${ }^{22}$ Shandong University (SDU), Jinan, Shandong, 250100, China

${ }^{23}$ Shandong Institute of Advanced Technology (SDIAT), Jinan, Shandong, 250100, China

${ }^{24}$ Jülich Supercomputing Centre and JARA-FAME, Research Centre Jülich, 52425 Jülich, Germany

${ }^{25}$ Institut für Experimentelle Teilchenphysik, Karlsruhe Institute of Technology (KIT), 76131 Karlsruhe, Germany

${ }^{26}$ Institut für Experimentelle und Angewandte Physik, Christian-Alberts-Universität zu Kiel, 24118 Kiel, Germany

${ }^{27}$ Instituto de Astrofísica de Canarias (IAC), 38205 La Laguna, and Departamento de Astrofísica, Universidad de La Laguna,38206 La

Laguna, Tenerife, Spain

${ }^{28}$ Laboratório de Instrumentação e Física Experimental de Partículas (LIP), 1649-003 Lisboa, Portugal

${ }^{29}$ National Chung-Shan Institute of Science and Technology (NCSIST), Longtan, Tao Yuan, 32546, Taiwan

${ }^{30}$ Centro de Investigaciones Energ eticas, Medioambientales y Tecnológicas (CIEMAT), 28040 Madrid, Spain

${ }^{31}$ Instituto de Física, Universidad Nacional Autónoma de México (UNAM), Ciudad de México, 01000 México 
${ }^{32}$ INFN Sezione di Milano-Bicocca, 20126 Milano, Italy

${ }^{33}$ Universitá di Milano-Bicocca, 20126 Milano, Italy

${ }^{34}$ NRNU MEPhI (Moscow Engineering Physics Institute), Moscow, 115409 Russia

${ }^{35}$ Southeast University (SEU), Nanjing, 210096, China

${ }^{36}$ Sodankylä Geophysical Observatory and Space Physics and Astronomy Research Unit, University of Oulu, 90014 Oulu, Finland

${ }^{37}$ INFN Sezione di Perugia, 06100 Perugia, Italy

${ }^{38}$ Universitá di Perugia, 06100 Perugia, Italy

${ }^{39}$ INFN Sezione di Pisa, 56100 Pisa, Italy

${ }^{40}$ INFN TIFPA, 38123 Povo, Trento, Italy

${ }^{41}$ Universitá di Trento, 38123 Povo, Trento, Italy

${ }^{42}$ Agenzia Spaziale Italiana (ASI), 00133 Roma, Italy

${ }^{43}$ INFN Sezione di Roma 1, 00185 Roma, Italy

${ }^{44}$ Universitá di Roma La Sapienza, 00185 Roma, Italy

${ }^{45}$ INFN Sezione di Roma Tor Vergata, 00133 Roma, Italy

${ }^{46}$ National Cheng Kung University, Tainan, 70101, Taiwan

${ }^{47}$ Academia Sinica Grid Center (ASGC), Nankang, Taipei, 11529, Taiwan

${ }^{48}$ Institute of Physics, Academia Sinica, Nankang, Taipei, 11529, Taiwan

${ }^{49}$ Physics Department and Center for High Energy and High Field Physics, National Central University (NCU), Tao Yuan, 32054,

Taiwan

${ }^{50}$ Space Research Laboratory, Department of Physics and Astronomy, University of Turku, 20014 Turku, Finland 